\title{
Damage Identification of Urban Overpass Based on Hybrid Neurogenetic Algorithm Using Static and Dynamic Properties
}

\author{
Hanbing Liu, Xianqiang Wang, Yafeng Gong, and Yubo Jiao \\ College of Transportation, Jilin University, Changchun 130025, China \\ Correspondence should be addressed to Yubo Jiao; jiaoyb@jlu.edu.cn
}

Received 10 March 2015; Revised 12 May 2015; Accepted 18 May 2015

Academic Editor: Anaxagoras Elenas

Copyright ( 2015 Hanbing Liu et al. This is an open access article distributed under the Creative Commons Attribution License, which permits unrestricted use, distribution, and reproduction in any medium, provided the original work is properly cited.

\begin{abstract}
Urban overpass is an important component of transportation system. Health condition of overpass is essential to guarantee the safe operation of urban traffic. Therefore, damage identification of urban overpass possesses important practical significance. In this paper, finite element model of left auxiliary bridge of Qianjin Overpass is constructed and vulnerable sections of structure are chosen as objects for damage recognition. Considering the asymmetry of Qianjin bridge, change rate of modal frequency and strain ratio are selected as input parameters for hybrid neurogenetic algorithm, respectively. Identification effects of damage location and severity are investigated and discussed. The results reveal that the proposed method can successfully identify locations and severities with single and multiple damage locations; its interpolation ability is better than extrapolation ability. Comparative analysis with $\mathrm{BP}$ neural network is conducted and reveals that the damage identification accuracy of hybrid neurogenetic algorithm is superior to BP. The effectiveness between dynamic and static properties as input variable is also analyzed. It indicates that the identification effect of strain ratios is more satisfactory than frequency ratio.
\end{abstract}

\section{Introduction}

Bridge structures are exposed to various external environments including vehicle, wind, and temperature. The security and durability of many bridges decline and damage appears, which will lead to unexpected collapse. Therefore, damage identification for these bridges has important practical significance $[1-3]$.

Traditional nondestructive evaluation- (NDE-) based methods such as ultrasonic and X-ray techniques have been widely applied for damage detection. However, the NDEbased damage detection approaches face the criticism that users have to know the damage vicinity in advance and damage prone areas have to be readily accessible in bridge monitoring [4]. To overcome the shortcomings of these local damage detection methods, overall identification method based on modal properties was put forward. Its theoretical background is that damage will modify the stiffness and mass of structure and then alter the modal data. Conversely, modal parameters can be regarded as damage indicators of structure. Most widely used modal parameters for damage identification include modal frequency, modal flexibility, modal strain energy, and modal curvature [5-8].

Frequency is the most easily obtained modal parameter [9-12]. With improvement of signal processing and analyzing, the identification precision of frequency becomes more favorable. Cawley and Adams proposed a nondestructive method to assess the integrity of structures using measurements of structural natural frequencies. The results have shown that it could detect, locate, and quantify damage [13]. Guo and Yi built the functional relationship between structural damage and change rate of frequency based on modal perturbation theory and successfully realized the damage identification of location and level [14]. Patil and Maiti put forward a multicrack damage identification method based on modal frequency for slender Bernoulli-Euler beams [15]. Kim and Stubbs presented a method to locate and estimate size of crack by using natural frequency changes; numerical simulation verified its feasibility [16].

In recent years, Artificial Neural Networks- (ANNs-) based damage identification methods have been widely utilized because of their excellent pattern recognition capacity 
[17-19]. Kao and Hung [20] presented a novel neural network-based approach for detecting structural damage by using two-step method. Fang et al. [21] proposed a backpropagation (BP) neural network-based damage identification method using frequency response functions as input data. Numerical simulation demonstrated that neural network can assess damage conditions with favorable accuracy. Mehrjoo et al. [22] presented a method for estimating the damage intensities of joints for truss bridge structures using a BP neural network. Bakhary et al. [23] developed a statistical approach to take into account the effect of uncertainties in developing ANNs model. However, ANNs have several drawbacks. Their convergence rate is very low and the chances of getting struck in local minima are relatively high [17]. Complementarily, genetic algorithm (GA) is able to find global optimal solution on a complicated optimization problem. Therefore, hybrid neurogenetic algorithm is promising choice for damage identification of bridge.

This paper is aimed at damage assessment of urban overpass, whose structural characteristics and mechanical properties are more complicated than traditional bridges. Taking advantages of calculation features of ANNs and GA, the initial weights and thresholds of BP neural networks are optimized by GA. The change rate of frequency and nodal strain before and after damage are chosen as input parameters, respectively. Comparative analysis is conducted to verify the superiority between BP and neurogenetic algorithm and also between dynamic property and static property.

\section{Theoretical Background}

2.1. Basic Theory of ANNs. ANNs are information processing systems which mimic the network structure of actual human brain [24]. It can process complex logic operations and achieve nonlinear mapping adaptively through learning. The process contains the forward propagation and back propagation. BP neural network is a widely used back-propagation network and consists of an input layer, hidden layers, and an output layer. The typical three-layer neural network is shown in Figure 1, in which successive layers are connected by adaptive interconnected weights. Training process of neural network is to minimize the objective function through adjusting the interconnected weights. The objective function is generally taken as the sum-squared error function [17], as shown in

$$
E=\sum_{k=1}^{p} \sum_{m=1}^{n}\left(O_{k m}-T_{k m}\right)^{2},
$$

where $O_{k m}$ and $T_{k m}$ are the actual and target output of $m$ th neuron in output layer for $k$ th training pattern, respectively. $n$ is the number of outputs and $p$ is the number of patterns. The calculation process of actual output in neural network with three layers is shown in Figure 2.

The design of suitable network is based on the selection of the number of hidden layers, number of neurons in the hidden layers, and the training algorithm. This paper adopts the BP neural network with one hidden layer, shown in Figure 1, as basic network architecture. The suitable number

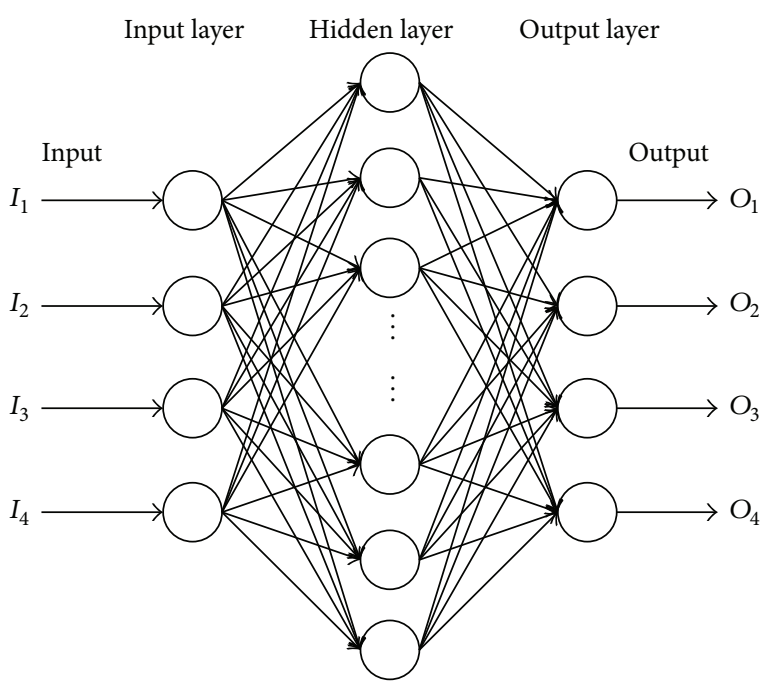

FIGURE 1: Architecture of three-layer BP neural network.

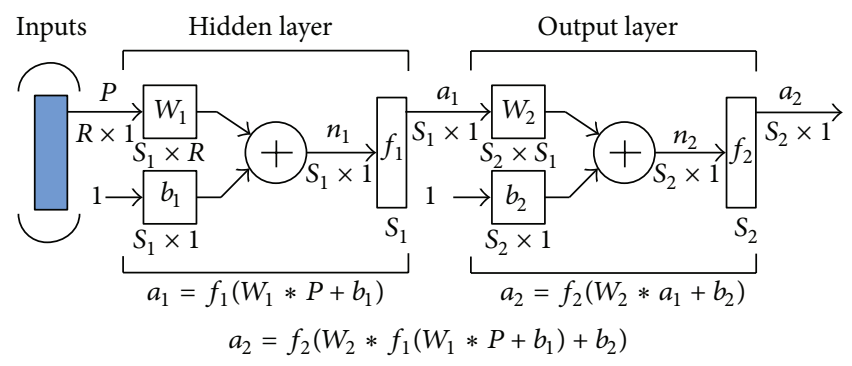

Figure 2: Calculation process of neural network.

of neurons in hidden layer is determined by multiple trial and errors in the standard neural network procedure. Considering the performance in minimizing mean square error (MSE) and accelerating convergence, neural networks with 10-13-4 topology (10 input layer neurons, 13 hidden layer neurons, and 4 output layer neurons) and 20-25-4 topology (20 input layer neurons, 25 hidden layer neurons, and 4 output layer neurons) are utilized to identify damage based on frequency and strain, respectively. In addition, the activation functions on hidden and output layers are sigmoid and linear function, respectively, while training algorithm adopts the LevenbergMarquardt (L-M) algorithm.

2.2. Basic Theory of GA. Genetic algorithm is adaptive probabilistic search algorithm for global optimization [25]. It is derived from the biological natural selection and genetic mechanisms. It is especially suitable for dealing with the complex and nonlinear problems which traditional search methods cannot resolve. Comparing with conventional optimization methods, the most highlighted advantage of GA is being not easy to trap in local optima due to many individuals (population) exploring in their search space [26].

In the optimization process of GA, the basic purpose is to maximize the fitness function by employing the three genetic operators called selection, crossover, and mutation. Firstly, 


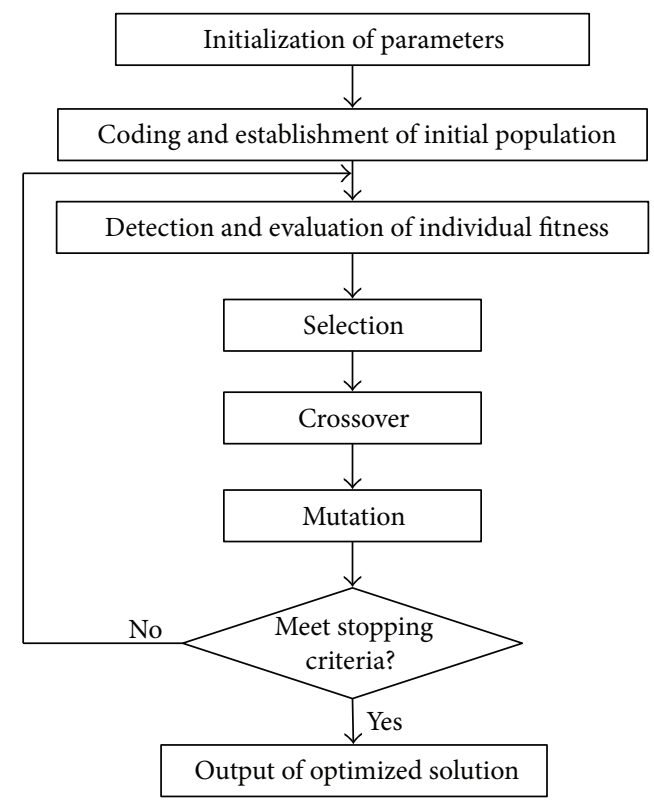

Figure 3: Processing flow of GA.

a population of individuals is initially generated at random and their fitness values are evaluated. Then, the selection operator selects a better set of individuals with higher fitness values in order to generate the next generation by the next two operators: crossover and mutation. In the crossover operator, two parents are selected randomly to breed two new offspring by exchanging different segments of the parents with each other. The mutation operator selects a random genome of a particular solution and alters its value to create new individual. Finally, with the evaluation of fitness for the new generation, the selection operator is utilized to select better parents for next generation. The above procedures are repeated for few generations until a given terminal criterion is satisfied. The basic calculation flow is shown in Figure 3.

The performance of GA is closely associated with coding, initial population, fitness function, genetic operation, and terminal condition. They are introduced in detail in the following.

(1) Coding. In GA, the individual in population is coded as a string of chromosome of given length $l$, and each string of chromosome represents a feasible solution to the optimization problem [27]. Because of the easy implementation to crossover and mutation operation, the binary code is usually used in GA. However, binary code makes the string of chromosome too long and fails to obtain high learning precision. It is incapable of coding parameter composed of the weights and thresholds of BP neural network. The real code is adopted in this paper to code the string of chromosome representing the weights and thresholds. Each position in chromosome denotes a weight or threshold of BP neural network.

(2) Initial Population. The production of GA begins with the initial population. Suppose that the population size is $N$.
The individuals in initial population are assigned values to their chromosomes randomly. The fitness of population is evaluated to guide the genetic operation. Each generation possesses the same size with initial population. The low number of the chromosomes will lead to searching a small part of the search space. According to the researches, a suitable population with 20-100 chromosomes can get best answers [28].

(3) Fitness Function. Each individual corresponds to different initial weights and thresholds of BP neural network. The fitness values of individuals represent the difference between actual outputs and expected outputs of BP neural network. The individual with bigger fitness is considered to have a better chance of survival. Fitness function is defined as follows:

$$
F=\frac{1}{1+E}
$$

where $E$ is the sum-squared error of BP neural network.

\section{(4) Genetic Operation}

(a) Selection. Roulette wheel selection is a selection operator based on the fitness of individuals. The selection probability of each individual as next generation is in proportion to its fitness. This probability of the $i$ th individual in population is calculated as follows:

$$
P_{i}=\frac{F_{i}}{\sum_{j=1}^{N} F_{j}},
$$

where $F_{i}$ is the fitness of $i$ th individual in population and $N$ is the population size. According to the Darwinian theory of survival of the fittest, it is more possible for the individual with better fitness in the current population to be selected and duplicated into the next generation.

(b) Crossover. Crossover operation creates offspring through the intersection of two parent chromosomes. The arithmetic crossover operator is adopted to perform crossover between two parent chromosomes coded as real variables. Suppose that two parent chromosome vectors are $N_{1}=$ $\left(x_{1}^{(1)}, x_{2}^{(1)}, \ldots, x_{m}^{(1)}\right)$ and $N_{2}=\left(x_{1}^{(2)}, x_{2}^{(2)}, \ldots, x_{m}^{(2)}\right)$ and two offspring are $N_{a}=\left(x_{1}^{(a)}, x_{2}^{(a)}, \ldots, x_{m}^{(a)}\right)$ and $N_{b}=\left(x_{1}^{(b)}, x_{2}^{(b)}, \ldots\right.$, $\left.x_{m}^{(b)}\right)$, respectively. $\left(\alpha_{1}, \alpha_{2}, \ldots, \alpha_{m}\right)$ are the random numerical values between 0 and 1 . The calculation process of nonuniform arithmetic crossover is defined as follows:

$$
\begin{aligned}
& x_{i}^{(a)}=\alpha_{i} x_{i}^{(1)}+\left(1-\alpha_{i}\right) x_{i}^{(2)}=x_{i}^{(2)}+\alpha_{i}\left(x_{i}^{(1)}-x_{i}^{(2)}\right), \\
& x_{i}^{(b)}=\alpha_{i} x_{i}^{(2)}+\left(1-\alpha_{i}\right) x_{i}^{(1)}=x_{i}^{(1)}+\alpha_{i}\left(x_{i}^{(2)}-x_{i}^{(1)}\right),
\end{aligned}
$$

where $i=1,2, \ldots, m$ and $m$ is the number of real variables in individual chromosome.

(c) Mutation. The nonuniform mutation operator is used to perform mutation in chromosomes. Suppose that parent chromosome $A=\left(x_{1}, x_{2}, \ldots, x_{k}, \ldots, x_{m}\right)$ produces mutation randomly at $k$ th variable and the variation range 


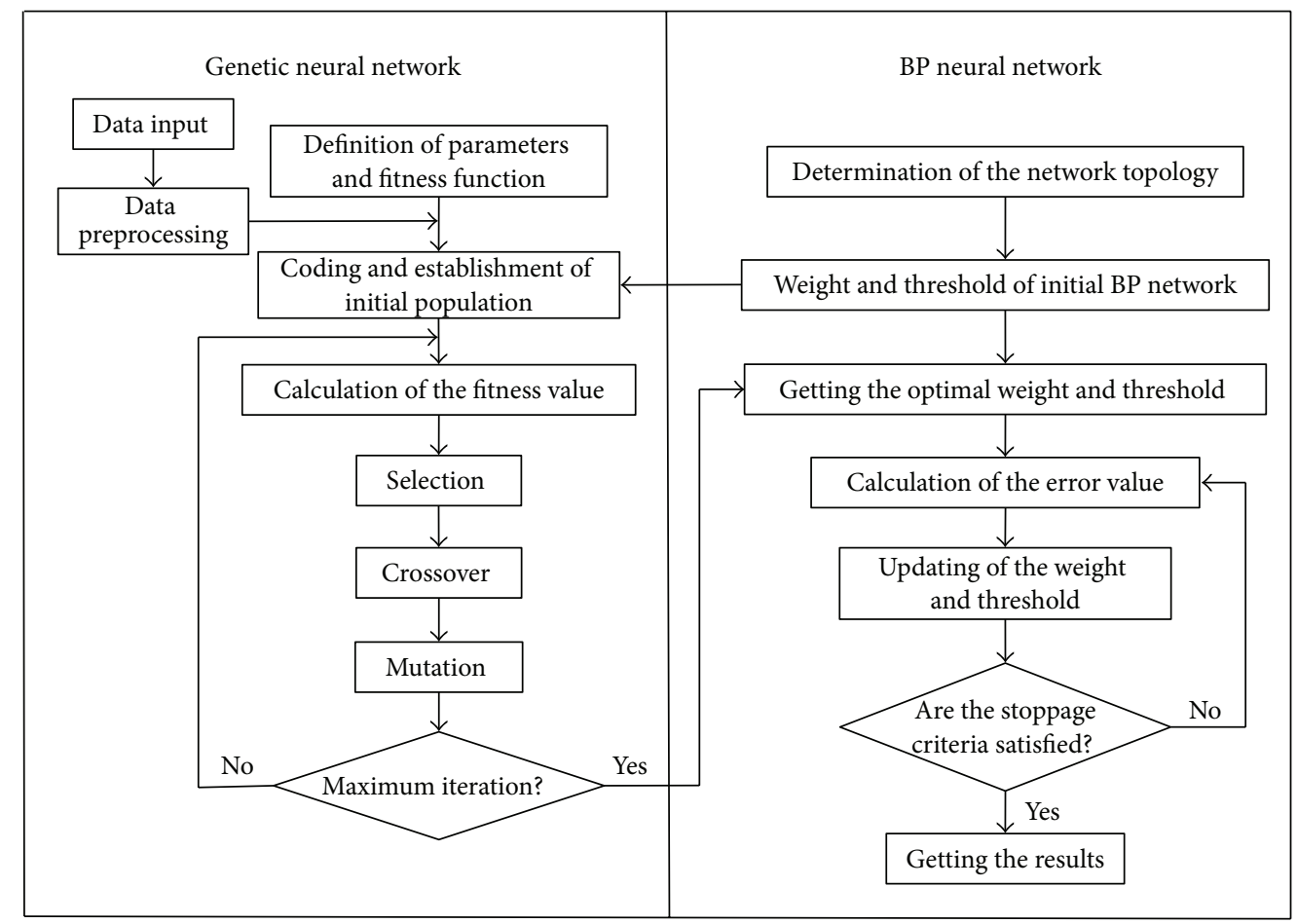

FIGURE 4: Calculation process of hybrid neurogenetic algorithm.

is $\left(a_{k}, b_{k}\right)$. The new offspring after mutation is $A^{\prime}=$ $\left(x_{1}, x_{2}, \ldots, x_{k}^{\prime}, \ldots, x_{m}\right), x_{k}^{\prime}$ is calculated according to

$$
x_{k}^{\prime}= \begin{cases}x_{k}+\Delta\left(t, b_{k}-x_{k}\right), & \text { rnd } \leq 0.5, \\ x_{k}-\Delta\left(t, x_{k}-a_{k}\right), & \text { rnd }>0.5\end{cases}
$$

$\Delta$ is defined as

$$
\Delta(t, y)=y \cdot r \cdot\left(1-\frac{t}{T}\right)^{\lambda}
$$

where rnd and $r$ are the random numbers between 0 and $1 ; t$ and $T$ are the current iteration time and the maximum number of iterations, respectively; $\lambda$ is a constant between 2 and 5, which controls the dependency of algorithm on iteration times.

(5) Terminal Condition. The optimization process of GA stops when the fitness of optimal individual satisfies the given critical value or the evolution reaches the maximum iteration. Generally, the maximum number of iterations is set between 100 and 500. In this paper, the maximum number of iterations is adopted as stoppage criteria.

2.3. Basic Theory of Hybrid Neurogenetic Algorithm. The prediction performance of BP neural network highly depends on the initial weights and thresholds of BP model. In this paper, the initial weights and thresholds are optimized by genetic algorithm. The combination of neural network and genetic algorithm can effectively overcome the shortcomings of neural network in order to realize the identification of damage location and severity for urban overpasses. The method of hybrid neurogenetic algorithm includes three steps: the determination of network structure, the optimization of genetic algorithm, and output prediction of BP neural network. Its basic process is shown in Figure 4.

The architectures of BP neural networks used to identify damage based on frequency and strain are determined as 1013-4 and 20-25-4, respectively. In order to avoid overfitting in neural network, the hidden layer neurons are defined based on the available data and multiple trials. Initial weights and thresholds are selected and coded as real variables in chromosomes. The optimization of GA is carried out with a population of 50 and the maximum number of iterations of 100. After the optimization for the initial weights and thresholds, BP neural network is trained with the setting parameters. The maximum number of iterations is 2000, tolerance error is $1.0 e-13$, and learning coefficients are set as 0.3 . In addition, a good preprocessing plays an important role in robustness and reliability of the networks. Therefore, the normalization for the input dada is carried out before using any databases. Hybrid neurogenetic algorithm makes the BP neural network more practical and accurate to identify damage in structure.

\section{Numerical Simulation}

3.1. Model Overview. Left auxiliary bridge of Qianjin Overpass in Changchun is a four-span continuous prestressed concrete box girder bridge. Each span has the length of $23 \mathrm{~m}$, and the height of girder is $1.3 \mathrm{~m}$. Schematic diagram for structure plan is shown in Figure 5. 


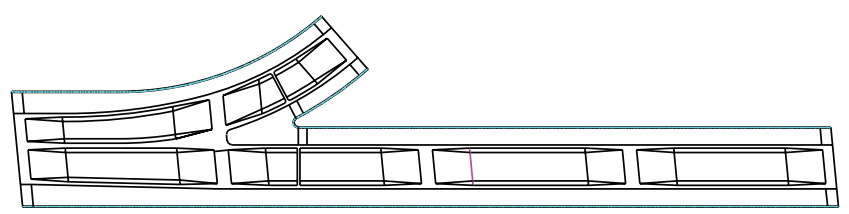

FIGURE 5: Structural plan.

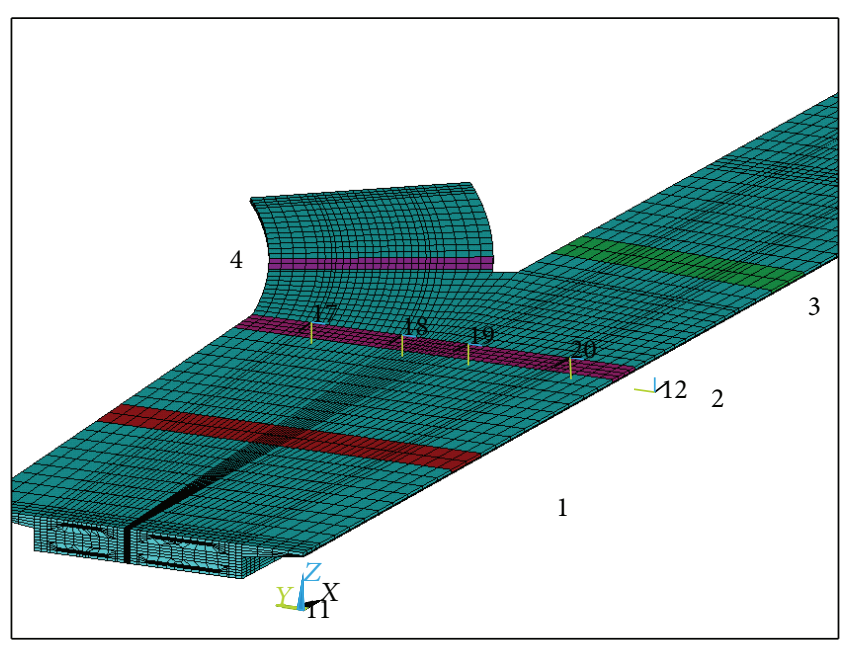

FIgURE 6: Determination of damaged sections.

3.2. Determination of Damage Sections. In consideration of the complexity of calculation model, it is unpractical to identify all elements of the structure, so vulnerable areas are chosen as objects for study in order to improve the computing efficiency. Bifurcation portion of bridge bears complicated bending-torsion coupling effect and becomes the emphasis area to identify damage. Therefore, research objects of this model include the middle section of the first span (section 1), the fulcrum section of the first span (section 2), and the middle section of the second span (section 3 and section 4). They are shown in Figure 6. The element damage is simulated through the reduction of elastic modulus and the percentage of reduction represents the damage extent.

\subsection{Determination of Parameters for Damage Identification}

(1) Input Parameters. Change rates of frequencies and nodal strains at characteristic sections are chosen as input parameters for hybrid neurogenetic algorithm. The dynamic input parameter can be calculated by

$$
\mathrm{FCR}_{i}=\frac{f_{u i}-f_{d i}}{f_{u i}}
$$

where $\mathrm{FCR}_{i}$ is the $i$ th-order frequency ratio and $f_{u i}$ and $f_{d i}$ represent $i$ th-order frequency of the model before and after damage, respectively.

The static input parameter can be expressed by

$$
\mathrm{SCR}_{j}=\left|\frac{S_{u j}-S_{d j}}{S_{u j}}\right|,
$$

where $\mathrm{SCR}_{j}$ is the strain ratio at node $j$ and $S_{u j}$ and $S_{d j}$ represent measured data of strains at node $j$ before and after damage, respectively.

Totally 20 nodes are selected as characteristic nodes, and strain ratios at these nodes are treated as input variables of hybrid neurogenetic algorithm. The characteristic nodes locate at the first mid-span, second mid-span, third midspan, and forth mid-span and also related fulcrum sections.

(2) Output Parameter. Damage levels of elements are simulated by the decline of element rigidity, defined as follows:

$$
\alpha_{i}=1-\frac{k_{i, d}}{k_{i, u}},
$$

where $i$ is the number of element, $\alpha_{i}$ is the damage level, $k_{i, d}$ is the element rigidity with damage, and $k_{i, u}$ is the element rigidity without damage.

The output parameter of hybrid neurogenetic algorithm is defined as follows:

$$
\text { output }=\left\{o_{1}, o_{2}, \ldots, o_{n}\right\}^{T} .
$$

For identification of damage location, $o_{i}=1$ represents that section $i$ has damage and $o_{i}=0$ represents that section $i$ is undamaged.

For identification of damage severity, $o_{i}$ represents damage level of section $i\left(\alpha_{i}\right)$.

\subsection{Damage Identification Based on Change Rate of \\ Frequency and Hybrid Neurogenetic Algorithm}

(1) Damage Identification with Single Damage Location. Taking damage identification of sections 1, 2, 3, and 4, for example, the levels of damage are considered in the range between $10 \%$ and $50 \%$, or six damage levels of $10 \%, 20 \%$, $30 \%, 35 \%, 40 \%$, and 50\%. 16 training samples are chosen with damage levels of $10 \%, 20 \%, 30 \%$, and $40 \%$, while 8 test samples with damage levels of $35 \%$ and $50 \%$ are used to check the damage identification performance. Testing cases are listed in Table 1.

Results of damage location identification are shown in Table 2. It indicates that hybrid neurogenetic algorithm can successfully identify the location of damage, and it has favorable generalization ability.

As for damage severity identification, the damage identification results are shown in Table 3, and the corresponding error curve and fitness curve are shown in Figures 7 and 8.

As can be seen from Table 3, the maximum error for testing samples with damage level $35 \%$ is $3.1 \%$, while it is $4.66 \%$ for damage level $50 \%$. It indicates that hybrid neurogenetic algorithm has better interpolation ability and relatively weaker extrapolation ability.

(2) Damage Identification with Multiple Damage Locations. Damage cases with multiple damage locations are listed in Table 4. For example, the case with damage levels of $20 \%-20 \%$ in damage sections 1 and 3 represents that the damage severity 
TABLE 1: Testing cases for damage identification with single damage location.

\begin{tabular}{lcc}
\hline $\begin{array}{l}\text { Testing } \\
\text { case }\end{array}$ & $\begin{array}{c}\text { Damage section } \\
\text { (corresponding } \\
\text { expected output) }\end{array}$ & $\begin{array}{c}\text { Damage severity } \\
\text { (corresponding } \\
\text { expected output) }\end{array}$ \\
\hline S1 & $1(1-0-0-0)$ & $35 \%(0.35-0-0-0)$ \\
S2 & $1(1-0-0-0)$ & $50 \%(0.5-0-0-0)$ \\
S3 & $2(0-1-0-0)$ & $35 \%(0-0.35-0-0)$ \\
S4 & $2(0-1-0-0)$ & $50 \%(0-0.5-0-0)$ \\
S5 & $3(0-0-1-0)$ & $35 \%(0-0-0.35-0)$ \\
S6 & $3(0-0-1-0)$ & $50 \%(0-0-0.5-0)$ \\
S7 & $4(0-0-0-1)$ & $35 \%(0-0-0-0.35)$ \\
S8 & $4(0-0-0-1)$ & $50 \%(0-0-0-0.5)$ \\
\hline
\end{tabular}

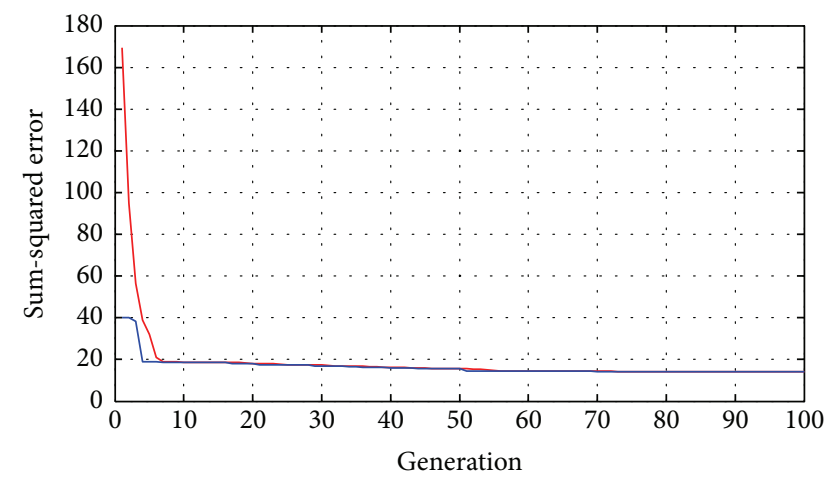

FIGURE 7: Error curve of damage severity identification with single damage location.

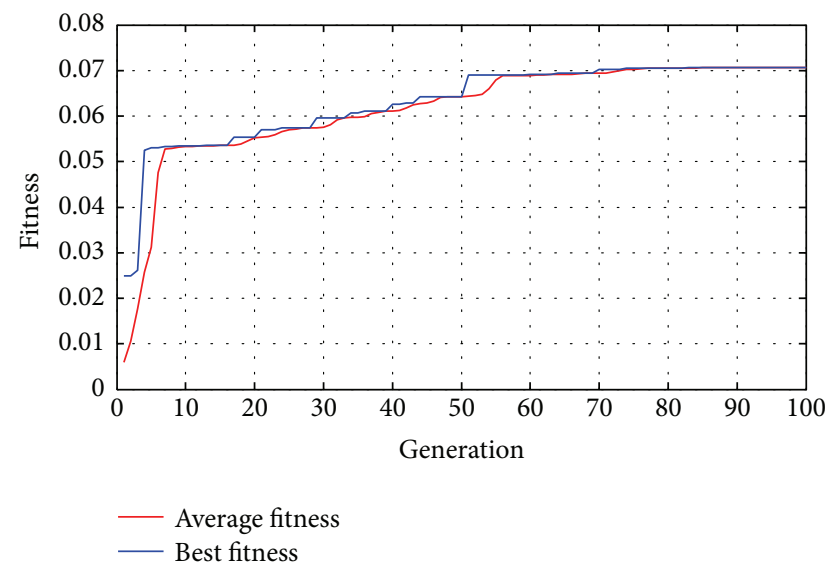

FIGURE 8: Fitness curve of damage severity identification with single damage location.

of section 1 is $20 \%$ and that of section 3 is also $20 \%$. Eight samples with damage levels of 20\%-30\%, 30\%-35\%, 35\%-35\%, and $40 \%-20 \%$ of sections 1 and 3 and 20\%-30\%, 30\%-35\%, $35 \%-35 \%$, and $40 \%-20 \%$ of sections 2 and 4 are selected as testing cases, as shown in Table 5, while the others are training samples.

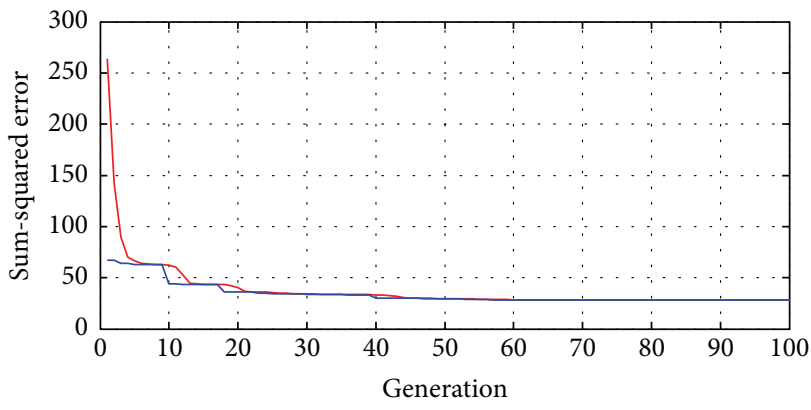

FIGURE 9: Error curve of damage severity identification with multiple damage locations.

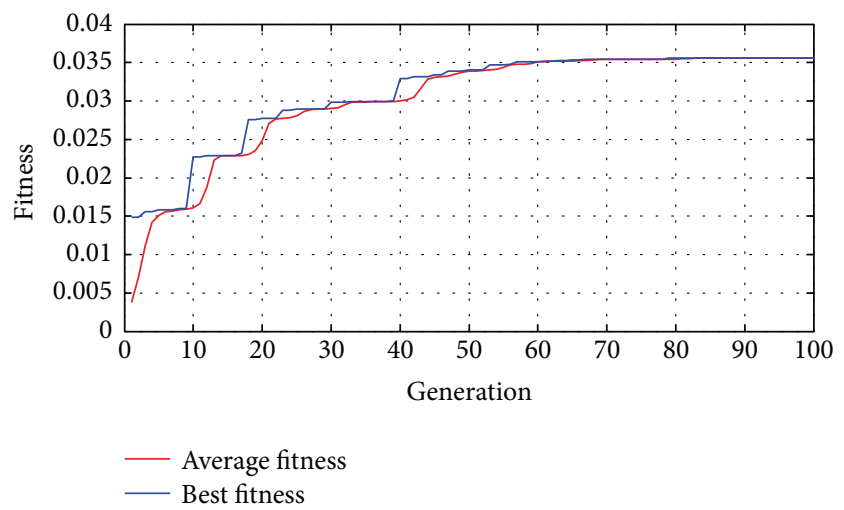

FIGURE 10: Fitness curve of damage severity identification with multiple damage locations.

Results of damage locations identification are listed in Table 6 . The results reveal that damage localization of hybrid neurogenetic algorithm with multi-damage locations is favorable.

The damage severity identification results with multiple locations are shown in Table 7, and the corresponding error curve and fitness curve are shown in Figures 9 and 10.

As can be seen from Table 7, hybrid neurogenetic algorithm can successfully identify the damage levels for multiple damage locations with favorable accuracy. The maximum relative error is $5 \%$.

\subsection{Damage Identification Based on Strain Ratio and Hybrid Neurogenetic Algorithm}

(1) Damage Identification with Single Damage Location. Training and test samples are the same with damage identification using frequency ratio. Results of damage location identification are listed in Table 8 . The results reveal that damage localization results are favorable.

The damage severity identification results are shown in Table 9. As can be seen from Table 9, the maximum error for samples with damage level $35 \%$ is $2.29 \%$, while it is $2.5 \%$ for damage level $50 \%$. It also indicates that hybrid neurogenetic algorithm has better interpolation ability and relatively weaker extrapolation ability. 
TABLE 2: Damage localization with single damage using frequency ratio.

\begin{tabular}{|c|c|c|c|c|c|c|c|c|}
\hline \multirow{2}{*}{ Output results } & \multicolumn{8}{|c|}{ Testing cases } \\
\hline & S1 & S2 & S3 & S4 & S5 & S6 & S7 & S8 \\
\hline \multirow{4}{*}{ Expected outputs } & 1 & 1 & 0 & 0 & 0 & 0 & 0 & 0 \\
\hline & 0 & 0 & 1 & 1 & 0 & 0 & 0 & 0 \\
\hline & 0 & 0 & 0 & 0 & 1 & 1 & 0 & 0 \\
\hline & 0 & 0 & 0 & 0 & 0 & 0 & 1 & 1 \\
\hline \multirow{4}{*}{ Actual outputs } & 1.004 & 1.003 & 0.000 & -0.004 & 0.000 & -0.003 & -0.001 & -0.001 \\
\hline & -0.004 & -0.003 & 1.000 & 1.005 & 0.000 & 0.003 & 0.001 & 0.001 \\
\hline & -0.001 & 0.001 & -0.001 & -0.003 & 1.001 & 1.001 & -0.001 & 0.002 \\
\hline & 0.001 & 0.000 & 0.000 & 0.002 & -0.001 & -0.001 & 1.000 & 0.998 \\
\hline
\end{tabular}

TABLE 3: Damage severity identification with single damage location using frequency ratio.

\begin{tabular}{|c|c|c|c|c|c|c|c|c|}
\hline \multirow{2}{*}{ Output results } & \multicolumn{8}{|c|}{ Testing cases } \\
\hline & $\mathrm{S} 1$ & S2 & S3 & S4 & S5 & S6 & S7 & S8 \\
\hline \multirow{4}{*}{ Expected outputs } & 0.35 & 0.5 & 0 & 0 & 0 & 0 & 0 & 0 \\
\hline & 0 & 0 & 0.35 & 0.5 & 0 & 0 & 0 & 0 \\
\hline & 0 & 0 & 0 & 0 & 0.35 & 0.5 & 0 & 0 \\
\hline & 0 & 0 & 0 & 0 & 0 & 0 & 0.35 & 0.5 \\
\hline \multirow{4}{*}{ Actual outputs } & 0.3453 & 0.5268 & 0.0002 & 0.0078 & 0.0014 & 0.0047 & -0.0016 & 0.0078 \\
\hline & 0.0034 & 0.0085 & 0.3503 & 0.5034 & -0.0024 & 0.0020 & -0.0002 & -0.0096 \\
\hline & -0.0047 & 0.0038 & -0.0015 & -0.0016 & 0.3391 & 0.5190 & -0.0007 & -0.0036 \\
\hline & -0.0050 & 0.0039 & 0.0048 & -0.0092 & 0.0024 & 0.0013 & 0.3541 & 0.5218 \\
\hline \multirow{4}{*}{ Relative errors (\%) } & 1.34 & 4.66 & & & \multirow{4}{*}{3.1} & \multirow{4}{*}{3.8} & & \\
\hline & & & 0.08 & 0.68 & & & & \\
\hline & & & & & & & & \\
\hline & & & & & & & 1.17 & 4.36 \\
\hline
\end{tabular}

TABLE 4: Cases for damage identification with multiple locations.

\begin{tabular}{ll}
\hline Damage section & Damage severity \\
\hline & $20 \%-20 \% ; 20 \%-30 \% ; 20 \%-35 \% ; 20 \%-40 \% ;$ \\
Sections 1 and 3 & $30 \%-20 \% ; 30 \%-30 \% ; 30 \%-35 \% ; 30 \%-40 \% ;$ \\
& $35 \%-20 \% ; 35 \%-30 \% ; 35 \%-35 \% ; 35 \%-40 \% ;$ \\
& $40 \%-20 \% ; 40 \%-30 \% ; 40 \%-35 \% ; 40 \%-40 \% ;$ \\
\hline & $20 \%-20 \% ; 20 \%-30 \% ; 20 \%-35 \% ; 20 \%-40 \% ;$ \\
Sections 2 and 4 & $30 \%-20 \% ; 30 \%-30 \% ; 30 \%-35 \% ; 30 \%-40 \% ;$ \\
& $35 \%-20 \% ; 35 \%-30 \% ; 35 \%-35 \% ; 35 \%-40 \% ;$ \\
& $40 \%-20 \% ; 40 \%-30 \% ; 40 \%-35 \% ; 40 \%-40 \% ;$ \\
\hline
\end{tabular}

Note: case with damage levels of $\mathrm{A} \%-\mathrm{B} \%$ in damage sections $\mathrm{C} 1$ and $\mathrm{C} 2$ represents that the damage severity of section $\mathrm{Cl}$ is $\mathrm{A} \%$ and that of section $\mathrm{C} 2$ is $\mathrm{B} \%$.

TABLE 5: Testing cases for damage identification with multiple damage location.

\begin{tabular}{lcc}
\hline $\begin{array}{l}\text { Testing } \\
\text { case }\end{array}$ & $\begin{array}{c}\text { Damage section } \\
\text { (corresponding } \\
\text { expected output) }\end{array}$ & $\begin{array}{c}\text { Damage severity } \\
\text { (corresponding expected output) }\end{array}$ \\
\hline M1 & 1 and 3 (1-0-1-0) & 20\%-30\% (0.2-0-0.3-0) \\
M2 & 1 and 3 (1-0-1-0) & $30 \%-35 \%(0.3-0-0.35-0)$ \\
M3 & 1 and 3 (1-0-1-0) & $35 \%-35 \%(0.35-0-0.35-0)$ \\
M4 & 1 and 3 (1-0-1-0) & $40 \%-20 \%(0.4-0-0.2-0)$ \\
M5 & 2 and 4 (0-1-0-1) & $20 \%-30 \%(0-0.2-0-0.3)$ \\
M6 & 2 and 4 (0-1-0-1) & $30 \%-35 \%(0-0.3-0-0.35)$ \\
M7 & 2 and 4 (0-1-0-1) & $35 \%-35 \%(0-0.35-0-0.35)$ \\
M8 & 2 and 4 (0-1-0-1) & $40 \%-20 \%(0-0.4-0-0.2)$ \\
\hline
\end{tabular}

TABLE 6: Damage localization of multi-damage locations using frequency ratio.

\begin{tabular}{lcccccccc}
\hline \multirow{2}{*}{ Output results } & \multicolumn{10}{c}{ Testing cases } \\
& M1 & M2 & M3 & M4 & M5 & M6 & M7 & M8 \\
\hline \multirow{5}{*}{ Expected outputs } & 1 & 1 & 1 & 1 & 0 & 0 & 0 & 0 \\
& 0 & 0 & 0 & 0 & 1 & 1 & 1 & 1 \\
& 1 & 1 & 1 & 1 & 0 & 0 & 0 & 0 \\
Actual outputs & 0 & 0 & 0 & 0 & 1 & 1 & 1 & 1 \\
\hline & 1.01 & 1.01 & 0.99 & 1.01 & 0.01 & 0.00 & 0.00 & 0.00 \\
& -0.02 & -0.01 & 0.01 & -0.01 & 0.99 & 1.00 & 1.00 & 1.00 \\
& 1.01 & 1.01 & 1.00 & 1.01 & 0.01 & 0.00 & 0.00 & 0.00 \\
& -0.01 & -0.01 & 0.00 & -0.01 & 0.99 & 1.00 & 1.00 & 1.00 \\
\hline
\end{tabular}

TABLE 7: Damage severity identification with multiple damage locations using frequency ratio.

\begin{tabular}{|c|c|c|c|c|c|c|c|c|}
\hline \multirow{2}{*}{ Output results } & \multicolumn{8}{|c|}{ Testing cases } \\
\hline & M1 & M2 & M3 & M4 & M5 & M6 & M7 & M8 \\
\hline \multirow{4}{*}{ Expected outputs } & 0.2 & 0.3 & 0.35 & 0.4 & 0 & 0 & 0 & 0 \\
\hline & 0 & 0 & 0 & 0 & 0.2 & 0.3 & 0.35 & 0.4 \\
\hline & 0.3 & 0.35 & 0.35 & 0.2 & 0 & 0 & 0 & 0 \\
\hline & 0 & 0 & 0 & 0 & 0.3 & 0.35 & 0.35 & 0.2 \\
\hline \multirow{4}{*}{ Actual outputs } & 0.21 & 0.31 & 0.34 & 0.41 & 0.00 & 0.00 & 0.00 & 0.00 \\
\hline & 0.00 & -0.01 & 0.01 & -0.02 & 0.20 & 0.30 & 0.35 & 0.40 \\
\hline & 0.30 & 0.35 & 0.34 & 0.21 & 0.00 & 0.00 & 0.00 & 0.00 \\
\hline & 0.02 & 0.01 & 0.00 & 0.00 & 0.31 & 0.35 & 0.35 & 0.20 \\
\hline \multirow{3}{*}{ Relative errors (\%) } & 5.00 & 3.33 & 2.85 & 2.50 & \multirow[b]{2}{*}{0} & \multirow[b]{2}{*}{0} & \multirow[b]{2}{*}{0} & \multirow[b]{2}{*}{0} \\
\hline & 0 & 0 & 285 & 500 & & & & \\
\hline & & & & & 3.33 & 0 & 0 & 0 \\
\hline
\end{tabular}


TABLE 8: Damage location identification with single damage using strain ratio.

\begin{tabular}{|c|c|c|c|c|c|c|c|c|}
\hline \multirow{2}{*}{ Output results } & \multicolumn{8}{|c|}{ Testing cases } \\
\hline & S1 & S2 & S3 & S4 & S5 & S6 & S7 & S8 \\
\hline \multirow{4}{*}{ Expected outputs } & 1 & 1 & 0 & 0 & 0 & 0 & 0 & 0 \\
\hline & 0 & 0 & 1 & 1 & 0 & 0 & 0 & 0 \\
\hline & 0 & 0 & 0 & 0 & 1 & 1 & 0 & 0 \\
\hline & 0 & 0 & 0 & 0 & 0 & 0 & 1 & 1 \\
\hline \multirow{4}{*}{ Actual outputs } & 1.0000 & 0.9999 & 0.0001 & -0.0002 & 0.0002 & 0.0009 & 0.0000 & 0.0000 \\
\hline & 0.0000 & 0.0000 & 1.0000 & 0.9999 & 0.0002 & -0.0005 & -0.0001 & -0.0001 \\
\hline & 0.0000 & 0.0000 & 0.0000 & -0.0001 & 1.0001 & 0.9997 & 0.0000 & 0.0000 \\
\hline & 0.0000 & 0.0000 & -0.0001 & 0.0001 & 0.0000 & -0.0003 & 1.0000 & 0.9998 \\
\hline
\end{tabular}

TABLE 9: Damage severity identification with single damage location using strain ratio.

\begin{tabular}{|c|c|c|c|c|c|c|c|c|}
\hline \multirow{2}{*}{ Output results } & \multicolumn{8}{|c|}{ Testing cases } \\
\hline & S1 & S2 & S3 & S4 & S5 & S6 & S7 & S8 \\
\hline \multirow{4}{*}{ Expected outputs } & 0.35 & 0.5 & 0 & 0 & 0 & 0 & 0 & 0 \\
\hline & 0 & 0 & 0.35 & 0.5 & 0 & 0 & 0 & 0 \\
\hline & 0 & 0 & 0 & 0 & 0.35 & 0.5 & 0 & 0 \\
\hline & 0 & 0 & 0 & 0 & 0 & 0 & 0.35 & 0.5 \\
\hline \multirow{4}{*}{ Actual outputs } & 0.342 & 0.5125 & -0.0006 & 0.0036 & -0.0004 & 0.0083 & 0.0032 & 0.0028 \\
\hline & 0.0005 & 0.0007 & 0.3526 & 0.5011 & 0.0001 & 0 & -0.0008 & -0.0012 \\
\hline & 0.0000 & -0.0001 & -0.0001 & -0.0002 & 0.3512 & 0.5023 & 0.0001 & 0.0002 \\
\hline & 0.0022 & 0.0036 & 0.0007 & -0.0034 & 0 & -0.0019 & 0.3468 & 0.5081 \\
\hline \multirow{4}{*}{ Relative errors (\%) } & 2.29 & 2.5 & & & & & & \\
\hline & & & 0.74 & 0.22 & & & & \\
\hline & & & & & 0.33 & 0.47 & & \\
\hline & & & & & & & 0.9 & 1.6 \\
\hline
\end{tabular}

(2) Damage Identification with Multiple Damage Locations. Results of damage locations identification are listed in Table 10. The results reveal that damage localization of hybrid neurogenetic algorithm with multi-damage locations is favorable.

The damage severity identification results with multiple locations are shown in Table 11.

As can be seen from Table 11, hybrid neurogenetic algorithm can successfully identify the damage levels of multidamage cases with favorable accuracy. The maximum relative error is $2.42 \%$.

\section{Comparative Analysis}

In order to verify the effectiveness of the proposed method in this paper, comparative analysis between hybrid neurogenetic algorithm and BP network is conducted.

Taking the damage identification of sections 1 and 3 using frequency ratio as input variable, for example, damage cases are shown in Table 12. Two samples with damage degrees of $20 \%-35 \%$ and $30 \%-40 \%$ are selected as testing cases, while the others are training samples. Corresponding damage identification results are listed in Table 13.

As can be seen from Table 13, the maximal relative error of hybrid neurogenetic algorithm and $\mathrm{BP}$ is $5 \%$ and $8.57 \%$, respectively. It reveals that the damage identification accuracy of hybrid neurogenetic algorithm is superior to BP.

As for the effect using static and dynamic properties as input variables, comparison between Tables 7 and 11 presents that the identification effect of strain ratios is more satisfactory than frequency ratio.

\section{Conclusions}

A hybrid neurogenetic algorithm-based method is proposed for damage identification of urban overpass. Change rate of frequency and strain ratio are treated as input parameters, respectively. The optimized weight and threshold are treated as the initial value of ANNs to identify the damage of bridge structure. Damage identification with single and multiple locations is used to verify the feasibility of the proposed method.

For both input parameters, numerical simulation results reveal that hybrid neurogenetic algorithm possesses favorable ability to identify the location of damage both for single damage case and multi-damage cases. For damage severity identification, the interpolation ability is better than extrapolation ability.

Comparative analysis with BP neural network indicates that maximal error of hybrid neurogenetic algorithm and BP 
TABLE 10: Damage location identification of multi-damage locations using strain ratio.

\begin{tabular}{|c|c|c|c|c|c|c|c|c|}
\hline \multirow{2}{*}{ Output results } & \multicolumn{8}{|c|}{ Testing cases } \\
\hline & M1 & M2 & M3 & M4 & M5 & M6 & M7 & M8 \\
\hline \multirow{4}{*}{ Expected outputs } & 1 & 1 & 1 & 1 & 0 & 0 & 0 & 0 \\
\hline & 0 & 0 & 0 & 0 & 1 & 1 & 1 & 1 \\
\hline & 1 & 1 & 1 & 1 & 0 & 0 & 0 & 0 \\
\hline & 0 & 0 & 0 & 0 & 1 & 1 & 1 & 1 \\
\hline \multirow{4}{*}{ Actual outputs } & 1.0003 & 1.0003 & 0.9998 & 1.0001 & 0.0000 & 0.0000 & 0.0000 & 0.0005 \\
\hline & -0.0001 & -0.0001 & 0.0001 & 0.0030 & 1.0001 & 1.0001 & 0.9999 & 1.0002 \\
\hline & 0.9999 & 0.9999 & 1.0002 & 1.0025 & 0.0001 & 0.0001 & 0.0000 & -0.0004 \\
\hline & -0.0002 & -0.0001 & -0.0001 & 0.0014 & 1.0000 & 1.0001 & 1.0000 & 0.9999 \\
\hline
\end{tabular}

TABLE 11: Damage severity identification result of multidamage using strain ratio as parameter.

\begin{tabular}{|c|c|c|c|c|c|c|c|c|}
\hline \multirow{2}{*}{ Output results } & \multicolumn{8}{|c|}{ Testing cases } \\
\hline & M1 & M2 & M3 & M4 & M5 & M6 & M7 & M8 \\
\hline \multirow{4}{*}{ Expected outputs } & 0.2 & 0.3 & 0.35 & 0.4 & 0 & 0 & 0 & 0 \\
\hline & 0 & 0 & 0 & 0 & 0.2 & 0.3 & 0.35 & 0.4 \\
\hline & 0.3 & 0.35 & 0.35 & 0.2 & 0 & 0 & 0 & 0 \\
\hline & 0 & 0 & 0 & 0 & 0.3 & 0.35 & 0.35 & 0.2 \\
\hline \multirow{4}{*}{ Actual outputs } & 0.205 & 0.302 & 0.349 & 0.397 & -0.001 & 0.000 & 0.000 & 0.000 \\
\hline & 0.000 & 0.000 & 0.000 & -0.001 & 0.203 & 0.301 & 0.349 & 0.410 \\
\hline & 0.307 & 0.350 & 0.348 & 0.200 & 0.000 & 0.000 & 0.000 & -0.003 \\
\hline & 0.000 & 0.000 & 0.000 & 0.003 & 0.307 & 0.348 & 0.348 & 0.203 \\
\hline \multirow{4}{*}{ Relative errors (\%) } & 2.39 & 0.64 & 0.42 & 0.64 & \multirow{3}{*}{1.69} & \multirow{3}{*}{0.21} & \multirow{3}{*}{0.37} & \multirow{3}{*}{2.41} \\
\hline & & & & & & & & \\
\hline & 2.17 & 0.02 & 0.62 & 0.17 & & & & \\
\hline & & & & & 2.42 & 0.43 & 0.55 & 1.71 \\
\hline
\end{tabular}

TABLE 12: Damage cases for comparative analysis.

\begin{tabular}{ll}
\hline Damage section & Damage severity \\
\hline & $20 \%-20 \% ; 20 \%-30 \% ; 20 \%-35 \% ; 20 \%-40 \% ;$ \\
Sections 1 and 3 & $30 \%-20 \% ; 30 \%-30 \% ; 30 \%-35 \% ; 30 \%-40 \% ;$ \\
& $35 \%-20 \% ; 35 \%-30 \% ; 35 \%-35 \% ; 35 \%-40 \% ;$ \\
& $40 \%-20 \% ; 40 \%-30 \% ; 40 \%-35 \% ; 40 \%-40 \% ;$ \\
\hline
\end{tabular}

TABLE 13: Identification results of hybrid neurogenetic algorithm and BP.

\begin{tabular}{lcccc}
\hline Output results & \multicolumn{4}{c}{ Methods } \\
& Hybrid neurogenetic algorithm & \multicolumn{2}{c}{ BP networks } \\
\hline \multirow{3}{*}{ Expected outputs } & 0.2 & 0.3 & 0.2 & 0.3 \\
& 0.35 & 0 & 0 & 0 \\
& 0 & 0.4 & 0.35 & 0.4 \\
Actual outputs & 0.21 & 0 & 0 & 0 \\
& 0.00 & 0.29 & 0.2 & 0.31 \\
& 0.34 & 0.40 & -0.02 & 0.01 \\
& -0.01 & 0.00 & 0.01 & -0.02 \\
\hline & 5 & 3.33 & 0 & 3.33
\end{tabular}

Errors (\%)

$\begin{array}{llll}2.86 & 0 & 8.57 \quad 5\end{array}$

is $5 \%$ and $8.57 \%$, respectively. Hybrid neurogenetic algorithm possesses more favorable results for damage identification.

As for using static and dynamic properties as input variables, the damage identification effect using strain ratio as input variable is more favorable. However, the frequency ratio is more convenient to obtain in practical application.

\section{Conflict of Interests}

The authors declare that there is no conflict of interests regarding the publication of this paper.

\section{Acknowledgments}

The authors express their appreciation for the financial support of National Natural Science Foundation of China under Grant nos. 51408258, 51378236, and 51278222, China Postdoctoral Science Foundation funded project (2014M560237), Fundamental Research Funds for the Central Universities and Science (JCKY-QKJC06), and Technology Development Program of Jilin Province (20140203002SF). The authors are very grateful for the reviewers and TMEE 2011.

\section{References}

[1] N. Kulprapha and P. Warnitchai, "Structural health monitoring of continuous prestressed concrete bridges using ambient thermal responses," Engineering Structures, vol. 40, pp. 20-38, 2012. 
[2] A. Rytter, Vibration based inspection of civil engineering structures [Ph.D. thesis], Department of Building Technology and Structural Engineering, Aalborg University, Aalborg, Denmark, 1993.

[3] J. V. A. dos Santos, C. M. M. Soares, C. A. M. Soares, and N. M. M. Maia, "Structural damage identification: influence of model incompleteness and errors," Composite Structures, vol. 62, no. 34, pp. 303-313, 2003.

[4] P. Lu, A statistical based damage detection approach for highway bridge structural health monitoring [Ph.D. thesis], Iowa State University, Ames, Iowa, USA, 2008.

[5] M. Chandrashekhar and R. Ganguli, "Damage assessment of structures with uncertainty by using mode-shape curvatures and fuzzy logic," Journal of Sound and Vibration, vol. 326, no. 3-5, pp. 939-957, 2009.

[6] A. K. Pandey, M. Biswas, and M. M. Samman, "Damage detection from changes in curvature mode shapes," Journal of Sound and Vibration, vol. 145, no. 2, pp. 321-332, 1991.

[7] Y. Xia, H. Hao, J. M. W. Brownjohn, and P.-Q. Xia, "Damage identification of structures with uncertain frequency and mode shape data," Earthquake Engineering and Structural Dynamics, vol. 31, no. 5, pp. 1053-1066, 2002.

[8] D. Ribeiro, R. Calçada, R. Delgado, M. Brehm, and V. Zabel, "Finite element model updating of a bowstring-arch railway bridge based on experimental modal parameters," Engineering Structures, vol. 40, pp. 413-435, 2012.

[9] O. S. Salawu, "Detection of structural damage through changes in frequency: a review," Engineering Structures, vol. 19, no. 9, pp. 718-723, 1997.

[10] M. A.-B. Abdo and M. Hori, "A numerical study of structural damage detection using changes in the rotation of mode shapes," Journal of Sound and Vibration, vol. 251, no. 2, pp. 227239, 2002.

[11] P. Z. Qiao, K. Lu, W. Lestari, and J. L. Wang, "Curvature mode shape-based damage detection in composite laminated plates," Composite Structures, vol. 80, no. 3, pp. 409-428, 2007.

[12] Q. Lu, G. Ren, and Y. Zhao, "Multiple damage location with flexibility curvature and relative frequency change for beam structures," Journal of Sound and Vibration, vol. 253, no. 5, pp. 1101-1114, 2003.

[13] P. Cawley and R. D. Adams, "The location of defects in structures from measurements of natural frequencies," Journal of Strain Analysis for Engineering Design, vol. 14, no. 2, pp. 49-57, 1979.

[14] G. H. Guo and W. J. Yi, "A numerical study on the damage assessment of a simply-supported beam on natural frequencies," Journal of Chongqing Jianzhu University, vol. 23, pp. 17-21, 2001.

[15] D. P. Patil and S. K. Maiti, "Detection of multiple cracks using frequency measurements," Engineering Fracture Mechanics, vol. 70, no. 12, pp. 1553-1572, 2003.

[16] J.-T. Kim and N. Stubbs, "Crack detection in beam-type structures using frequency data," Journal of Sound and Vibration, vol. 259, no. 1, pp. 145-160, 2003.

[17] B. Sahoo and D. Maity, "Damage assessment of structures using hybrid neuro-genetic algorithm," Applied Soft Computing Journal, vol. 7, no. 1, pp. 89-104, 2007.

[18] C. Na, S.-P. Kim, and H.-G. Kwak, "Structural damage evaluation using genetic algorithm," Journal of Sound and Vibration, vol. 330, no. 12, pp. 2772-2783, 2011.
[19] J. J. Lee, J. W. Lee, J. H. Yi, C. B. Yun, and H. Y. Jung, "Neural networks-based damage detection for bridges considering errors in baseline finite element models," Journal of Sound and Vibration, vol. 280, no. 3-5, pp. 555-578, 2005.

[20] C. Y. Kao and S.-L. Hung, "Detection of structural damage via free vibration responses generated by approximating artificial neural networks," Computers and Structures, vol. 81, no. 28-29, pp. 2631-2644, 2003.

[21] X. Fang, H. Luo, and J. Tang, "Structural damage detection using neural network with learning rate improvement," Computers and Structures, vol. 83, no. 25-26, pp. 2150-2161, 2005.

[22] M. Mehrjoo, N. Khaji, H. Moharrami, and A. Bahreininejad, "Damage detection of truss bridge joints using Artificial Neural Networks," Expert Systems with Applications, vol. 35, no. 3, pp. 1122-1131, 2008.

[23] N. Bakhary, H. Hao, and A. J. Deeks, "Damage detection using artificial neural network with consideration of uncertainties," Engineering Structures, vol. 29, no. 11, pp. 2806-2815, 2007.

[24] M. T. Hagan, H. B. Demuth, and M. H. Beale, Neural Network Design, University of Colorado Bookstore, 2002.

[25] H.-B. Liu and Y.-B. Jiao, "Application of genetic algorithmsupport vector machine (GA-SVM) for damage identification of bridge," International Journal of Computational Intelligence and Applications, vol. 10, no. 4, pp. 383-397, 2011.

[26] A. Rabiei, H. Sayyad, M. Riazi, and A. Hashemi, "Determination of dew point pressure in gas condensate reservoirs based on a hybrid neural genetic algorithm," Fluid Phase Equilibria, vol. 387, pp. 38-49, 2015.

[27] A. Azadeh, S. F. Ghaderi, S. Tarverdian, and M. Saberi, "Integration of artificial neural networks and genetic algorithm to predict electrical energy consumption," Applied Mathematics and Computation, vol. 186, no. 2, pp. 1731-1741, 2007.

[28] D. E. Goldberg, Genetic Algorithm in Search, Optimization and Machine Learning, Addison-Wesley, Harlow, UK, 1989. 


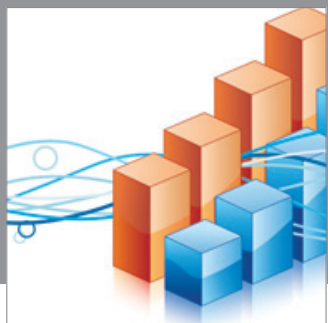

Advances in

Operations Research

mansans

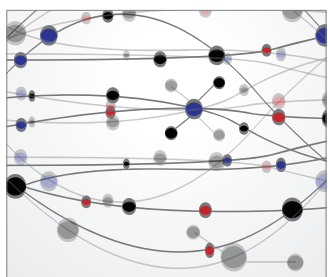

The Scientific World Journal
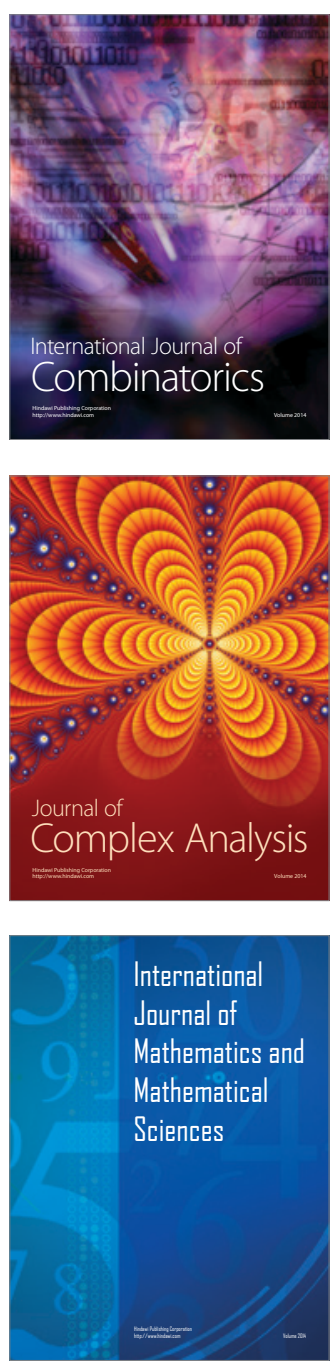
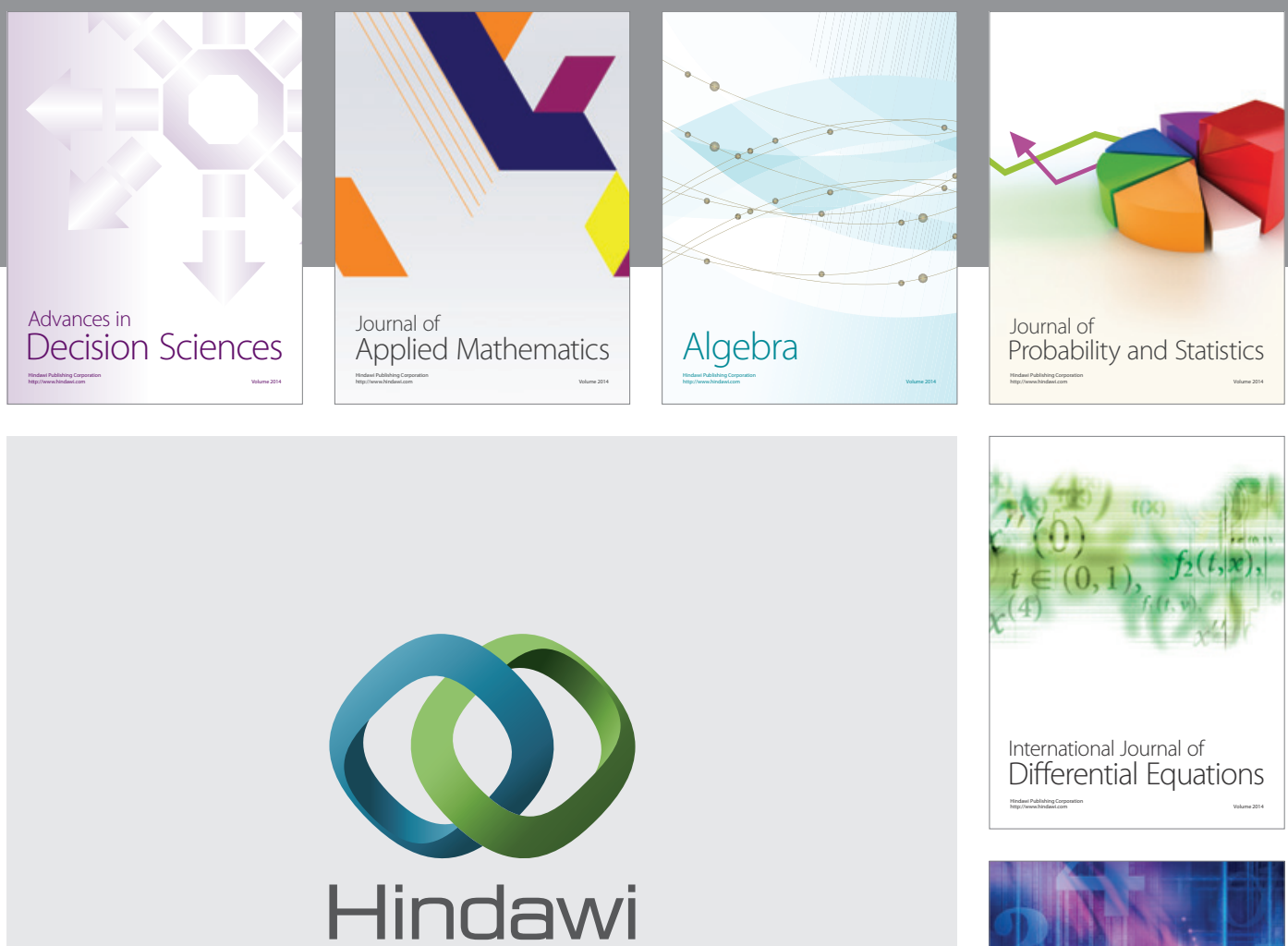

Submit your manuscripts at http://www.hindawi.com
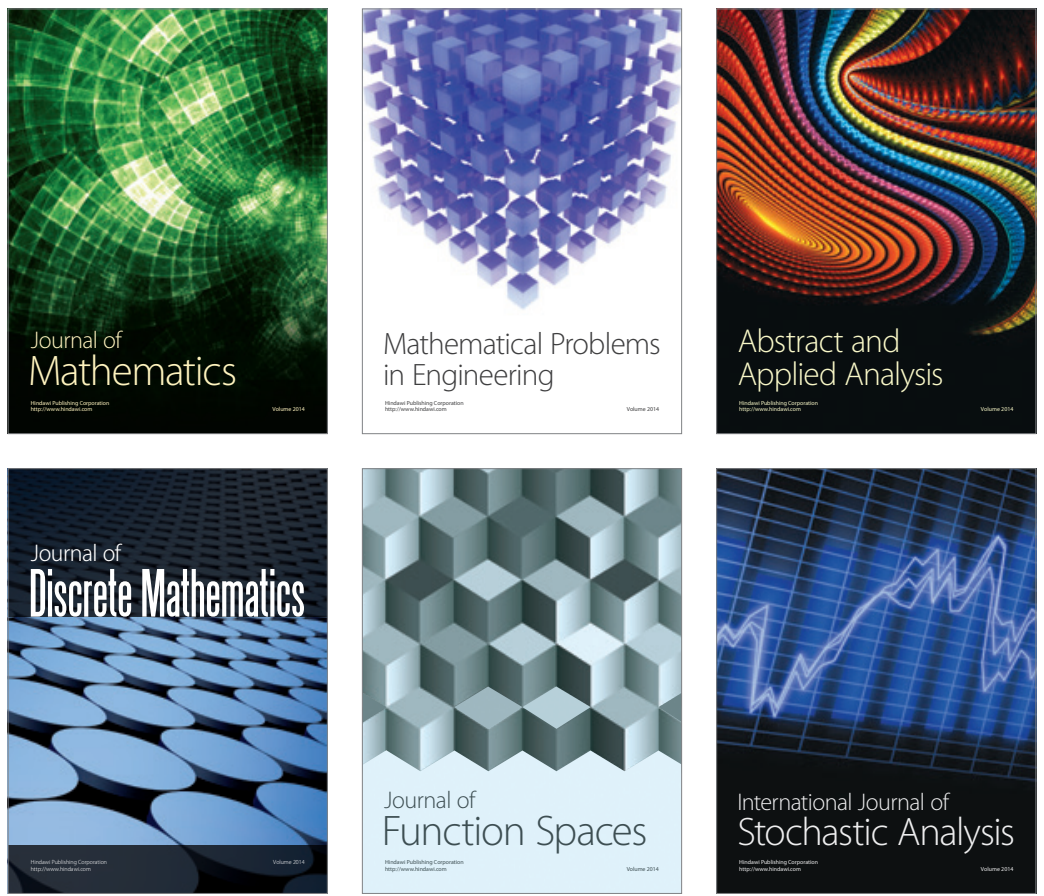

Journal of

Function Spaces

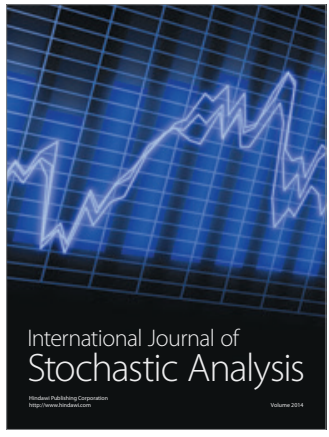

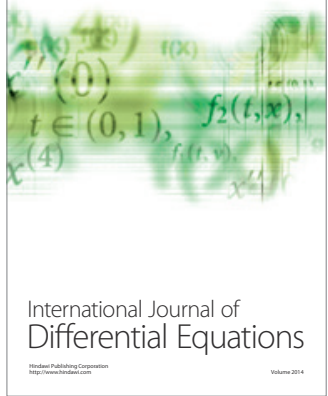
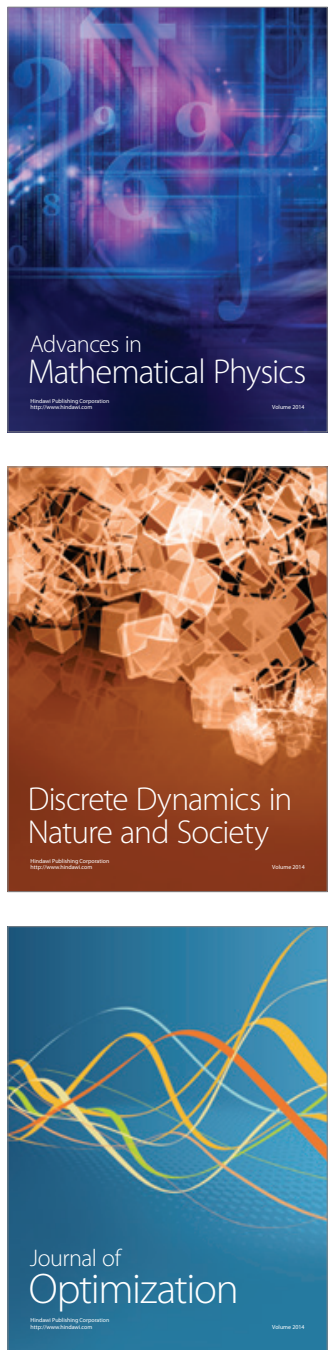